\title{
Modelling gap junctions in a neural field model Amanda Elvin*
}

\author{
Address: Institute of Information and Mathematical Sciences, Massey University, Albany, Auckland, New Zealand \\ Email: Amanda Elvin* - A.J.Elvin@massey.ac.nz \\ * Corresponding author
}

from Sixteenth Annual Computational Neuroscience Meeting: CNS*2007

Toronto, Canada. 7-12 July 2007

Published: 6 July 2007

BMC Neuroscience 2007, 8(Suppl 2):P73 doi:I0.I I86/I47|-2202-8-S2-P73

() 2007 Elvin; licensee BioMed Central Ltd.

We study a nonlinear, one-dimensional neural field model based upon a partial integro-differential equation, that is used to model spatial patterns in working memory. Through the application of Fourier transforms to the PIDE, steady states of spatially localised areas of high activity can be represented by solutions of a fourth order ODE. Recent research has shown a high density of gap junctions in areas of the brain that experience epileptic events. We extend the model by including a diffusion-like term to model gap junctions and derive a sixth order ODE which we use to investigate changes in the dynamics of spatially localised solutions. We find that symmetric homoclinic orbits to a zero steady state exist for a wide area of parameter space. Numerical work shows families of solutions are destroyed as the strength of the term modelling gap junctions increases. 